\title{
Mitochondrial tRNA import in Trypanosoma brucei is independent of thiolation and the Rieske protein
}

\author{
ZDENĚK PARIS, ${ }^{1,2}$ MARY ANNE T. RUBIO, ${ }^{3,4}$ JULIUS LUKEŠ, ${ }^{1,2}$ and JUAN D. ALFONZO ${ }^{3,4}$ \\ ${ }^{1}$ Biology Centre, Institute of Parasitology, Czech Academy of Sciences, 37005 Ceské Budejovice (Budweis), Czech Republic \\ ${ }^{2}$ Faculty of Sciences, University of South Bohemia, 37005 Ceské Budejovice (Budweis), Czech Republic \\ ${ }^{3}$ Department of Microbiology, Ohio State Biochemistry Program, The Ohio State University, Columbus, Ohio 43210, USA \\ ${ }^{4}$ OSU Center for RNA Biology, The Ohio State University, Columbus, Ohio 43210, USA
}

\begin{abstract}
Due to a complete lack of the tRNA genes in the mitochondrial genome of Trypanosoma brucei, all tRNAs needed for mitochondrial translation have to be imported into the organelle from the cytosol. A previous study showed that the modified nucleotide $s^{2} U$ could act as a negative determinant for mitochondrial tRNA import in another kinetoplastid, Leishmania tarentolae. We have investigated whether the same type of cytosolic control for tRNA retention exists in $T$. brucei. Based on Northern analysis with subcellular RNA fractions and in vitro import assays, we demonstrate that silencing of the cysteine desulfurase, TbNfs (TblscS), the key enzyme in tRNA thiolation $\left(s^{2} U\right)$ and Fe-S cluster formation in vivo, has no effect on tRNA partitioning. This observation is especially surprising in light of a recent report suggesting that in $L$. tropica the Rieske Fe-S protein is an essential component of the RNA import complex (RIC). In line with the above observation, we also show that down-regulation of the Rieske protein by RNA interference, similar to the TbNfs knockdowns, has no effect on import. The data presented here supports the view that in $T$. brucei: (1) $s^{2} U$ is not a negative determinant for tRNA import; (2) the Rieske protein is not an essential component of the import machinery, and (3) since the Rieske protein is essential for respiration and maintenance of inner mitochondrial membrane potential, neither process plays a critical role in tRNA import. We therefore suggest that the $T$. brucei import machinery differs substantially from what has been described in Leishmania.
\end{abstract}

Keywords: T. brucei; tRNA import; 2-thiolation; RIC; Rieske; Fe-S cluster

\section{INTRODUCTION}

In a majority of eukaryotes, most of the ATP produced in cells is the result of mitochondrial respiration, which depends on the synthesis and assembly of respiratory complexes. The bulk of the respiratory proteins are encoded in the nucleus, translated in the cytoplasm, and subsequently imported into the mitochondria where together with a few mitochondria-encoded subunits constitute the mature, fully functional complexes. Therefore, nominally, mitochondrial respiration requires both active cytosolic protein import systems and protein synthesizing machinery within the mitochondrial matrix.

Although mitochondrial protein synthesis is invariably essential, many mitochondrial genomes retained only an

Reprint requests to: Juan D. Alfonzo, Department of Microbiology, Ohio State Biochemistry Program and OSU Center for RNA Biology, The Ohio State University, Columbus, 43210 OH, USA; e-mail: alfonzo.1@ osu.edu; fax: (614) 292-8120.

Article published online ahead of print. Article and publication date are at http://www.rnajournal.org/cgi/doi/10.1261/rna.1589109. incomplete set of tRNAs insufficient for translation (Salinas et al. 2008). These organisms have thus evolved mechanisms to import tRNAs across the mitochondrial membranes. To date, tRNA import systems have been described in a number of diverse organisms including the ciliate Tetrahymena (Suyama 1986; Rusconi and Cech 1996a,b), the apicomplexan Toxoplasma (Esseiva et al. 2004), the kinetoplastid flagellates Trypanosoma and Leishmania (Simpson et al. 1989; Mottram et al. 1991; Hancock et al. 1992), plants (Marechal-Drouard et al. 1988), yeast (Martin et al. 1979; Rinehart et al. 2005), and most recently mammals (Rubio et al. 2008).

Two main general mechanisms have been described for tRNA import: one mechanism originally described in yeast for the import of tRNA ${ }^{\text {Lys }}$ is similar to protein import (Tarassov et al. 1995a). This system requires an electrochemical potential across the mitochondrial inner membrane, and with the exception of the mitochondrial outer membrane protein MOM72, all other protein import components play a role in tRNA ${ }^{\text {Lys }}$ import (Martin et al. 1979; Tarassov and Martin 1996; Tarassov et al. 1995a). 
Import also requires the in vivo association of the tRNA with the precursor mitochondrial tRNA synthetase and aminoacylation of the tRNA by the cognate cytosolic synthetase (Schmitz and Lonsdale 1989; Tarassov et al. 1995b), and the metabolic enzyme enolase to help delivery of the imported tRNA/synthetase complex to the import machinery on the mitochondrial surface (Entelis et al. 2006).

Second, and perhaps the most common mechanism for tRNA import, was originally described in Leishmania and is now also found in most other organisms ranging from kinetoplastids to humans (Schneider and MarechalDrouard 2000; Salinas et al. 2008). This mechanism is independent of the protein import pathway and can be efficiently reproduced in vitro in the absence of cytosolic factors (Mahapatra et al. 1994; Yermovsky-Kammerer and Hajduk 1999; Rubio et al. 2000, 2008); however, it may still be influenced by their presence. For example, in Trypanosoma brucei, cytosolic translation elongation factor 1a (eEF1a) plays a key role as a specificity determinant for some imported tRNAs (Bouzaidi-Tiali et al. 2007). More recently, the import of tRNA ${ }^{\mathrm{Gln}}$ into yeast mitochondria was shown to occur in vitro in the absence of added cytosolic factors (Rinehart et al. 2005). Therefore, Saccharomyces cerevisiae contains two pathways for tRNA import, a feature that is thus far unique to this organism (Rinehart et al. 2005).

What constitutes a conserved tRNA determinant or recognition element for organellar import has also been under debate. In Leishmania, the mature tRNA is the in vivo substrate for import (Kapushoc et al. 2000). Although a full-length tRNA is the preferred substrate in vitro (Rubio et al. 2000), smaller substrates representing regions of the full-length tRNA could be efficiently imported and apparently contain useful import information (Mahapatra et al. 1998). Furthermore, in L. tarentolae, Suzuki and coworkers showed that tRNA modifications, specifically the presence of 2-thiouridine $\left(s^{2} \mathrm{U}\right)$ in the anticodon of tRNA ${ }^{\text {Glu }}$, could serve as a negative determinant for import. In their system, only the unmodified tRNA (not containing $s^{2} \mathrm{U}$ ) was the substrate for import in vivo and in vitro, while the modified version was retained in the cytoplasm (Kaneko et al. 2003).

In recent years, numerous protein factors have been implicated as additional requirements for import of tRNAs into mitochondria. In plants, for instance, the voltagedependent anion channel (VDAC) in the outer membrane was shown to play a crucial role for import in vitro (Salinas et al. 2006). However, the homologous protein is dispensable for import in T. brucei (Pusnik et al. 2009). Despite sharing a common basic mechanism, not requiring the protein import pathway, a majority of tRNA import systems may thus possess nuances that set them apart and that may reflect the polyphyletic origin of mitochondrial tRNA import machineries (Schneider and Marechal-Drouard 2000).
To date, one of the most studied import systems is that of Leishmania, the causative agent of human leishmaniasis. Adhya and coworkers have identified a putative RNA import complex (RIC) in L. tropica, which contains a number of proteins known to serve alternative functions in respiration, introducing the idea of a "moonlighting" function for these proteins (Mukherjee et al. 2007). The core import complex contains six essential factors required for both in vivo and in vitro import: $\alpha$ subunit of the $\mathrm{F}_{1}$-ATP synthase; subunit $6 \mathrm{~b}$ and the Rieske protein of the respiratory complex III; two proteins with no similarities to any other proteins in the database; and finally, subunit 6 of the respiratory complex IV (Mukherjee et al. 2007).

In this report, we have investigated whether $s^{2} U$ is a negative determinant for import of tRNAs into the single mitochondrion of $T$. brucei, a generally close relative of Leishmania, hoping to establish commonalities between the two systems. Using RNA interference (RNAi), we have knocked down two T. brucei proteins, the homologs of which play a role in Leishmania tRNA import: TbNfs (formery TbIscS2) and the Rieske protein, both previously shown to be essential for the Fe-S cluster assembly and respiration in T. brucei, respectively (Smid et al. 2006). We show that although the inhibition of T. brucei TbNfs led to a concomitant decrease of tRNA ${ }^{\text {Glu }}$ thiolation, it had no effect on the distribution of this tRNA species in vivo or in vitro, suggesting that $\mathrm{s}^{2} \mathrm{U}$ is not a negative determinant for tRNA import in this protist. Furthermore, our findings also question the conservation of the Rieske protein as a general and essential tRNA import factor, since down-regulation of this protein causes dramatic decrease of mitochondrial membrane potential and cytochrome-dependent respiration, yet without causing any effect on the tRNA distribution in vivo or in vitro. Taken together, the data presented here support the view that thiolation, respiration, membrane potential, and most importantly, the Rieske protein are not essential components of the T. brucei mitochondrial tRNA import pathway.

\section{RESULTS}

\section{TbNfs is essential for growth of $T$. brucei}

TbNfs is the master desulfurase responsible for both Fe-S cluster formation and tRNA thiolation. The putative function of the $s^{2} U$ modification as a negative determinant for tRNA import into the T. brucei mitochondria can be tested in cells depleted for TbNfs, as this protein is known to be essential for thiolation at U34 in tRNA ${ }^{\text {Glu }}$ (Kambampati and Lauhon 2000, 2003; Mihara et al. 2002; Nilsson et al. 2002; Nakai et al. 2004; Ikeuchi et al. 2006). We therefore took advantage of the previously described $T$. brucei procyclic cells, in which TbNfs (=TbIscS2) can be inducibly down-regulated (Smid et al. 2006). With these cells, inhibition of growth is apparent $3 \mathrm{~d}$ after the induction 
of RNAi, and the cells virtually stopped growing by day 5 (Fig. 1A). The RNAi-induced cells showed no recovery even after $8 \mathrm{~d}$ of cultivation (Fig. 1; data not shown). The extent of TbNfs silencing was evaluated using specific polyclonal anti-TbNfs antibodies, which revealed almost complete elimination of the targeted protein by day 3 of RNAi induction (Fig. 1B). This observation leads to the conclusion that TbNfs is essential for growth of procyclic T. brucei.

\section{$s^{2} U$ is not a determinant for tRNA import into the $T$. brucei mitochondrion}

To determine whether TbNfs has a role in tRNA import, levels of several tRNAs were measured in mitochondria isolated from the noninduced and RNAi-induced TbNfs knockdown cells. Day 3, after the addition of tetracycline to induce RNAi, was selected for subsequent experiments, as the growth of the induced cells decreases after this
A

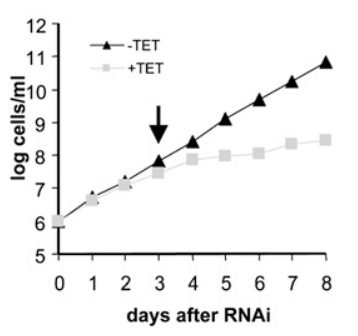

B

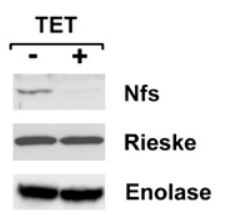

c

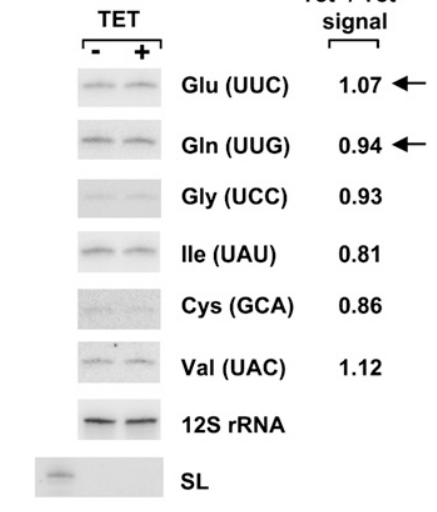

FIGURE 1. Effects of TbNfs RNAi on parasite growth. (A) Growth curves of the noninduced (TET-) (triangles) and RNAi-induced (TET+) (squares) knockdowns for TbNfs. Cell densities were measured using the Burker cell chamber. The $y$-axis is labeled by a log scale and represents the products of measured cell densities and the total dilutions. The arrow indicates sampling time point for latter experiments. (B) The effect of RNAi on the TbNfs protein levels was analyzed by Western blots with extracts from the noninduced $(-)$ and RNAi-induced $(+)$ cells. Cells were collected $3 \mathrm{~d}$ after RNAi induction. Each lane was loaded with total protein from $\sim 5 \times 10^{6}$ cells, and polyclonal anti-TbNfs antibodies were used for detection. Similar Western blots with anti-enolase and anti-Rieske protein antibodies were used as loading controls. $(C)$ Northern analysis of mitochondrial tRNAs purified from noninduced (-) and RNAi-induced (+) TbNfs cells. RNAs were visualized with radioactively labeled oligonucleotide probes specific for tRNA ${ }^{\text {Glu }}$, $\mathrm{tRNA}^{\mathrm{Gln}}$, $\mathrm{tRNA}^{\text {Gly }}, \mathrm{RNA}^{\mathrm{Ile}}, \mathrm{tRNA}^{\mathrm{Cys}}$, and tRNA ${ }^{\mathrm{Val}}$. Amounts of the individual tRNAs after RNAi induction were calculated as the ratio of the noninduced (Tet-) to induced (Tet+) signals, after normalization against the $12 \mathrm{~S}$ mitoribosomal RNA. The numbers indicate differences between the noninduced and induced cells, where a ratio of 1 indicates no differences between the two samples. (Arrows) Thiolated tRNAs. Cytosolic spliced leader (SL) RNA was used as a control for mitochondrial RNA purity. time point (Fig. 1A), while the TbNfs protein is virtually eliminated (Fig. 1B). After day 3 the culture still remains in proper physiological condition as determined by light microscopy (data not shown), which effectively excludes the influence of possible secondary phenotypic effects.

Two different approaches were used to investigate the role of the 2-thiouridine modifications in tRNA import. RNA isolated from organelles purified from the noninduced and induced TbNfs cells were analyzed by Northern blotting using a panel of oligonucleotide probes specific for either thiolated or nonthiolated tRNAs (Fig. 1C). The former class was represented by $\mathrm{tRNA}^{\text {Glu }}$ and $\mathrm{tRNA}^{\mathrm{Gln}}$, while four nonthiolated tRNAs were assayed (tRNA ${ }^{\text {Gly }}$, $\mathrm{tRNA}^{\mathrm{Ile}}$, $\mathrm{tRNA}^{\mathrm{Cys}}$, and $\mathrm{tRNA}^{\mathrm{Val}}$ ). The transcript levels of $12 \mathrm{~S}$ mitochondrial rRNA should be unaltered by the interference against $\mathrm{TbNfs}$ and thus served as a reference for calculation of relative abundances of the examined tRNA species. Prior to Northern analysis for tRNA distribution, subcellular RNA fractions were routinely tested for compartment cross-contamination by hybridization with a probe against the spliced leader (SL) RNA, which is confined to the cytosol. Indeed, no SL RNA signal was detected in the organellar RNA fractions while a clear signal was seen in the cytosolic fractions, indicating that the mitochondrial fractions had negligible levels of cytosolic contamination (Fig. 1C).

Surprisingly, quantification of the import of all tRNAs revealed no major differences between the nonthiolated and thiolated tRNA species (Fig. 1C). For individual tRNAs, differences between their presence in mitochondria isolated from the noninduced and RNA-induced cells ranged from $81 \%$ to $112 \%$ (or a ratio of 0.81 to 1.12 when the noninduced signal is divided by the induced for each tRNA). The mitochondrial localization did not correlate with the thiolation status of the molecules. A slight increase in the levels of tRNA ${ }^{\text {Glu }}$ was observed upon RNAi induction of TbNfs; however, this increase was deemed insignificant in that a slight decrease was documented for the other thiolated tRNA (tRNA ${ }^{\text {Gln }}$ ) examined (Fig. 1C). These experiments lead to the conclusion that $s^{2} U$ formation in tRNA is not a negative determinant for in vivo mitochondrial import in T. brucei.

To further substantiate the results described above, an in vitro import assay was performed following a previously described protocol (Rubio et al. 2000). First, we tested the import of native $\mathrm{tRNA}^{\mathrm{Glu}}$ and compared its import behavior with a similar sample that was pretreated with hydrogen peroxide to remove the $s^{2} U$ group. To ensure dethiolation, the $\mathrm{H}_{2} \mathrm{O}_{2}$-treated tRNA was separated in an (N-acryloylamino) phenyl-mercuric chloride (APM) gel (Crain et al. 2002). Both the $\mathrm{H}_{2} \mathrm{O}_{2}$-treated and untreated native $\mathrm{tRNA}^{\text {Glu }}$ species were then 5 '-labeled. Increasing concentrations of radioactively labeled dethiolated or thiolated tRNA ${ }^{\text {Glu }}$ were then used in import assays by incubating increasing concentrations of either substrate with 
A

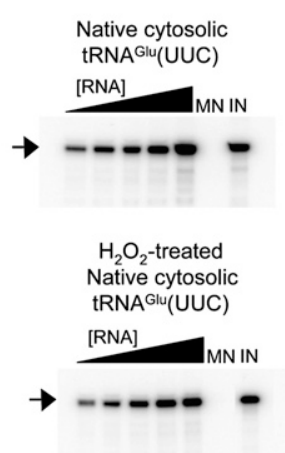

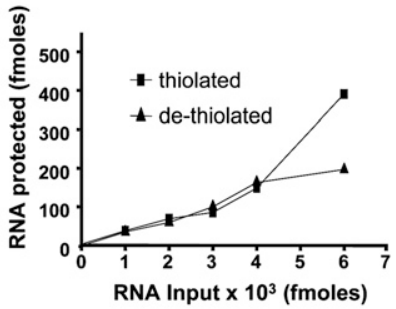

FIGURE 2. In vitro import of native thiolated and dethiolated tRNA $^{\text {Glu }}$. (A) Native tRNA ${ }^{\text {Glu }}$ was purified by oligo-affinity as described in the Materials and Methods. The resulting tRNA was end-labeled and used for in vitro import assays into isolated mitochondria (top). Prior to import, thiolation was confirmed by APM-Northern blot, which specifically detects thiolated species (data not shown). A portion of the same native tRNA fraction was treated with $\mathrm{H}_{2} \mathrm{O}_{2}$ to remove the sulfur and then used in a similar in vitro import assay (bottom). In both panels, the black triangle denotes increasing tRNA concentrations used in the assay. MN refers to a tRNA treated with micrococcal nuclease in the absence of mitochondria to show that the tRNA is not inherently resistant to the nuclease treatment. This lane is also used as a background control. IN refers to an untreated tRNA input lane used as a marker and also as a standard for quantification. The position of the full-length tRNA after gel electrophoresis (see Materials and Methods) is indicated with an arrow. $(B)$ The values obtained from $A$ were used to calculate the fmoles of tRNA protected in the assay, which was plotted versus input tRNA in fmoles.

Percoll gradient-purified mitochondria in the presence of ATP (Fig. 2). Reactions in which tRNAs were incubated in the import buffer but in the absence of mitochondria or micrococcocal nuclease served as markers during electrophoresis, as well as quantification standards. Reactions in which tRNAs were incubated in the presence of nuclease but in the absence of mitochondria served as a negative control for import. These experiments showed that regardless of whether or not a given tRNA is thiolated, it is efficiently imported into the mitochondrion in vitro (Fig. 2).

We also performed similar reactions with in vitro radioactively labeled transcripts representing either tRNA ${ }^{\mathrm{Glu}}$ or tRNA $^{\text {Ile }}$ (a nonthiolated tRNA) comparing mitochondria purified from tetracycline induced and noninduced RNAi TbNfs cells (Fig. 3A,B). We found that, analogous to the in vivo situation, import is not affected by the elimination of the cysteine desulfurase TbNfs (Fig. $3 \mathrm{C})$. Both tRNAs are imported in vitro with comparable efficiencies. Taken together, we can conclude that neither knockdowns are shown.
A

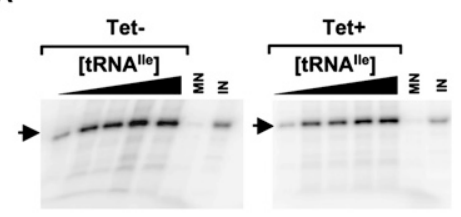

B

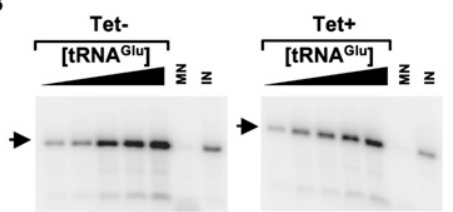

TbNfs nor the presence of $s^{2} \mathrm{U}$ in the tRNA play significant roles as tRNA import determinants in vivo or in vitro.

\section{Rieske is essential for growth but not for mitochondrial tRNA import in $T$. brucei}

It was recently proposed that the Rieske protein is an essential component of the RIC complex in L. tropica (Mukherjee et al. 2007). This Fe-S cluster containing subunit of the respiratory complex III is essential for respiration and also for maintenance of the membrane potential across the mitochondrial inner membrane. In light of our observation that down-regulation of TbNfs had no effect on tRNA import into the T. brucei mitochondrion, and the known requirement of $\mathrm{TbNfs}$ for overall Fe-S cluster assembly, it is difficult to reconcile our findings with the idea that the Rieske protein could also be essential for tRNA import in T. brucei. To test this proposal further, we have generated clonal cell lines for the inducible down-regulation of the Rieske protein expression by RNAi (Horváth et al. 2005). Since the cell growth decreases at day 4 following RNAi induction (Fig. 4A), this time point was selected for further experiments. As in the case of TbNfs knockdowns, no resistance against RNAi was observed even in cells cultivated for a prolonged period of time. Western analysis using specific antibodies showed a dramatic decrease of the Rieske protein levels upon the addition of tetracycline (Fig. 4B).

Next, the mitochondrial RNAs isolated from the noninduced cells, as well as from cells $4 \mathrm{~d}$ after RNAi induction, were subjected to Northern analysis with the same set of oligonucleotide probes used to analyze both nonthiolated

FIGURE 3. In vitro import of tRNA ${ }^{\text {Ile }}$ and tRNA ${ }^{\text {Glu }}$ transcripts into mitochondria from the TbNfs RNAi cells. Increasing concentrations of in vitro transcribed radioactively labeled tRNA $^{\text {Ile }}(A)$ and tRNA ${ }^{\text {Glu }}(B)$ were incubated with constant concentration of Percoll-purified mitochondria from the noninduced $(-)$ and RNAi-induced (+) TbNfs knockdowns. The black triangle denotes increasing concentrations of tRNA used in the assay. MN is the micrococcal nuclease control as described in Fig. 2, and IN is the input quantification control. The position of the full-length tRNA after gel electrophoresis (see Materials and Methods) is indicated with an arrow. (C) Graph of the results from $A$ and $B$, where pmoles of tRNA protected upon import into the mitochondrion are plotted versus pmoles of input used in the assay. Import reactions with mitochondria obtained from noninduced $(-)$ and RNAi-induced (+) TbNfs 
A
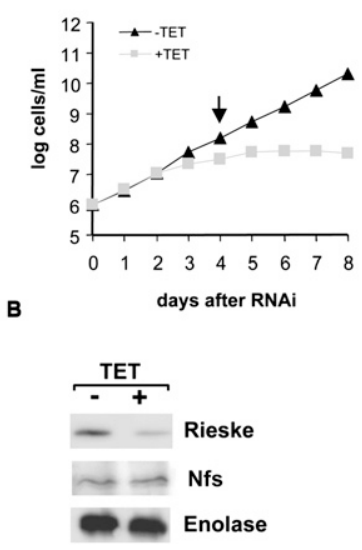

FIGURE 4. Effects of Rieske protein RNAi on parasite growth and tRNA localization in vivo. $(A)$ Growth curves of the noninduced (TET - ) (triangles) and RNAi-induced (TET+) (squares) knockdowns for the Rieske protein. Cell densities were measured using the Burker cell chamber. The $y$-axis is labeled by a log scale and represents the products of measured cell densities and the total dilutions. The arrow indicates sampling time point for latter experiments. $(B)$ The effect of RNAi on the Rieske protein levels was analyzed by Western blots with extracts from the noninduced (-) and RNAi-induced (+) cells. Cells were collected $4 \mathrm{~d}$ after RNAi induction. Each lane was loaded with total protein from $\sim 5 \times 10^{6}$ cells, and polyclonal anti-Rieske protein antibodies were used for detection. Similar Western blots with antienolase and anti-TbNfs antibodies were used as loading controls. $(C)$ Northern blot analysis of mitochondrial tRNAs purified from the noninduced ( $(-)$ and RNAi-induced $(+)$ Rieske protein cells. RNAs were visualized with radioactively labeled oligonucleotide probes specific for tRNA ${ }^{\text {Glu, }}$, tRNA ${ }^{\text {Gln }}$, tRNA ${ }^{\text {Gly }}$, RNA ${ }^{\text {Ile }}$, tRNA ${ }^{\text {Cys }}$, and tRNA $^{\text {Val }}$. Amounts of the individual tRNAs, after RNAi induction, were calculated as the ratio of noninduced (Tet-) to induced (Tet+) signals, after normalization against the $12 \mathrm{~S}$ mitoribosomal RNA. The numbers indicate differences between the noninduced and induced cells, where a ratio of 1 indicates no differences between the two samples. (Arrows) Thiolated tRNAs. Cytosolic spliced leader (SL) RNA was used as a control for mitochondrial RNA purity.

and thiolated tRNAs. As before, probes against the SL RNA and $12 \mathrm{~S}$ rRNA were used to assess sample purity and served as loading controls, respectively (Fig. 4C). These results showed no differences between the import behavior of the tRNAs tested, including the nonthiolated and/or thiolated species (Fig. 4C). In fact, both thiolated species (tRNA ${ }^{\text {Glu }}$ and $\mathrm{tRNA}^{\mathrm{Gln}}$ ) were slightly up-regulated in the RNAiinduced organelles (by $10 \%$ and $11 \%$, respectively); however, a similar up-regulation was observed with some of the nonthiolated tRNAs (Fig. 4C). Although we cannot formally exclude the possibility that the levels of tRNA detected are not due to tRNAs that were already present in the mitochondrion prior to the effect of RNAi, these experiments still suggest that the Rieske protein may play no role in mitochondrial tRNA import in T. brucei in vivo. Our results are also consistent with the observation that the downregulation of TbNfs has no effect on tRNA distribution.

To substantiate the findings above, we have also performed similar in vitro import experiments as in the pre- vious section but with mitochondria isolated from the noninduced and RNAi-induced Rieske knockdowns. The import efficiency directly correlates with the increasing amounts of input radiolabeled tRNAs, and the curves are virtually the same for the in vitro import into mitochondria purified from the noninduced and RNAi-induced cells. In both cases, a plateau is reached at about 400 pmol of input tRNAs in the reaction. These experiments showed that, like in the in vivo situation, in vitro tRNA import is not affected by the elimination of the Rieske protein (Fig. 5), which was proposed to be an essential subunit of the RIC in L. tropica.

\section{DISCUSSION}

Most eukaryotic cells contain an extensive degree of intracellular compartmentalization that allows subcellular organelles to keep distinct metabolic processes separated, often as a means to prevent unwanted reactions. In kinetoplastid flagellates, for example, recent studies have shown that compartmentalization of the glycolytic enzymes prevents accumulation of toxic glycolytic intermediates in the cytoplasm (Haanstra et al. 2008). What leads to intracellular compartmentalization is an extensive network of membrane systems, which are the foundations of intracellular architecture. Mitochondria and various types of plastids are unique membrane-bound intracellular compartments that usually contain their own genome, which encodes a variable number of protein-coding genes essential for organellar functions. However, these genomes are dynamic and throughout evolution have relegated a number of functions to the nucleus via gene transfer and gene loss. Indeed, a wide majority of mitochondrial proteins are encoded in the nucleus, synthesized in the cytoplasm and subsequently imported into the organelle. Remarkably, compartmentalization and the loss of mitochondrial genetic material frequently led to the disappearance of tRNA genes from mitochondrial genomes. This has in turn driven the evolution of mechanisms for the import of tRNAs from the cytoplasm to enable translation of the protein coding mRNAs still encoded in mitochondria (Salinas et al. 2008). Currently, however, the factors and/or mechanisms that control tRNA import are not fully understood.

Despite differences in the number and types of factors shown to be associated with tRNA import in various organisms, tRNAs can mechanistically be imported in two different ways: either via the protein import pathway (e.g., coimport with cytosolic aminoacyl-tRNA synthetase) (Tarassov et al. 1995b) or via a pathway independent from protein import that does not require cytosolic factors (Rubio et al. 2000). Future studies may reveal additional pathways. However, the disparate nature of the import mechanisms suggests that tRNA import evolved independently in different systems (Schneider and MarechalDrouard 2000). Differences among various systems may 
A

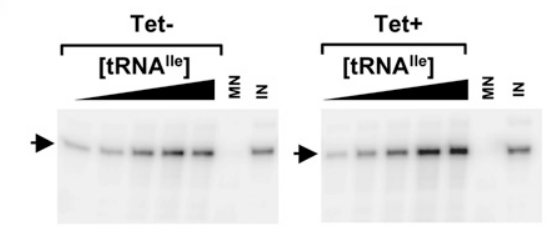

B

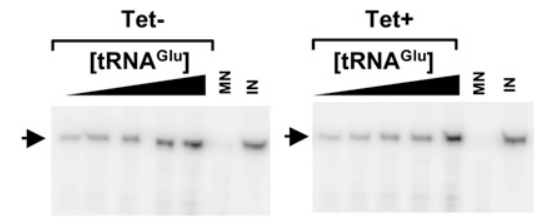

C

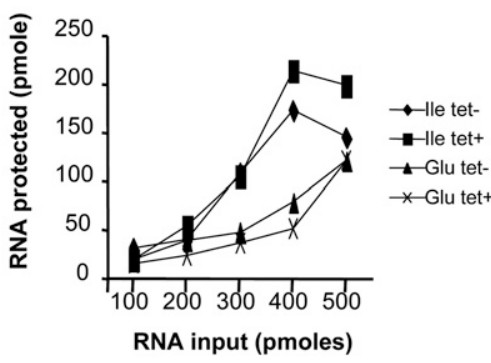

FIGURE 5. In vitro import of tRNA ${ }^{\text {Ile }}$ and $\mathrm{tRNA}^{\mathrm{Glu}}$ transcripts into mitochondria from the Rieske RNAi cells. Increasing concentrations of in vitro transcribed radioactively labeled tRNA $^{\text {Ile }}(A)$ and tRNA ${ }^{\text {Glu }}(B)$ were incubated with constant concentration of Percoll-purified mitochondria from the noninduced $(-)$ and RNAi-induced (+) Rieske protein knockdowns. The black triangle denotes increasing concentrations of tRNA used in the assay. MN is the micrococcal nuclease control as described in Fig. 2, and IN is the input quantification control. The position of the full-length tRNA after gel electrophoresis (see Materials and Methods) is indicated with an arrow. (C) Graph of the results from $A$ and $B$, where pmoles of RNA protected upon import into the mitochondrion are plotted versus pmoles of input used in the assay. - and + indicate import of the respective tRNAs into mitochondria from the noninduced and RNAi-induced $(+)$ cells as shown.

also suggest import as a dynamic process that has adapted to intracellular environmental changes provided by a particular organism.

Among the most studied tRNA import pathways are those of kinetoplastids, where a number of in vitro and/or in vivo determinants for import have been described (Salinas et al. 2008). It was reported that thiolation of tRNAs in the cytoplasm of $L$. tarentolae acted as a negative determinant for import of tRNA ${ }^{\text {Glu }}$ (Kaneko et al. 2003); however, the degree of phylogenetic conservation of this mechanism has not been explored further. In addition, different tRNA sequence determinants for import have been reported even within similar systems (Lima and Simpson 1996). Specific sequences in the D-arm and/or

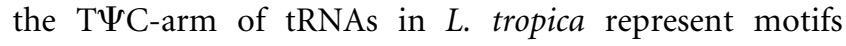
determining in vivo localization (Mahapatra et al. 1994). However, the presence of these sequences and their evolutionary conservation do not correlate with tRNA localization in L. tarentolae (Suyama et al. 1998).

One feature common to all tRNA import systems described so far is the requirement for ATP, which has led to a foray into the bioenergetic requirements of import in various organisms (Salinas et al. 2008). However, different groups have described different bioenergetic requirements. While an absolute requirement of mitochondrial membrane potential for tRNA import has been postulated by some groups (Yermovsky-Kammerer and Hajduk 1999; Bhattacharyya and Adhya 2004), we and others have questioned such a requirement (Nabholz et al. 1999; Rubio et al. 2000). Membrane potential is definitely essential for import of tRNA ${ }^{\text {Lys }}$ into the yeast mitochondria, where several fea- tures of the protein import pathway are required (Tarassov et al. 1995a); yet membrane potential is dispensable in vitro for a second tRNA import pathway recently described for tRNA ${ }^{\text {Gln }}$ import in yeast (Rinehart et al. 2005).

In terms of the involvement of protein factors in tRNA import, the VDAC is important in plants (Salinas et al. 2006), but establishing its specific role will require further exploration. In light of our knowledge of the tRNA ${ }^{\text {Lys }}$ import in yeast, the placement of the RIC among the inner mitochondrial membrane proteins in L. tropica is not unprecedented (Mukherjee et al. 2007). However, the composition of the RIC is certainly surprising, as it is allegedly composed of a number of proteins that also constitute respiratory complexes, such as the Rieske protein, and have been proposed to perform double duty as tRNA import factors in the context of RIC (Mukherjee et al. 2007).

In an attempt to find commonalities among the various kinetoplastid systems, and prompted by the availability of RNAi in T. brucei, we explored the use of thiolation as a conserved negative determinant for import in vivo in these ancestral flagellates. To this end, we have generated conditional RNAi knockdown cell lines of TbNfs and showed that like in all other organisms described so far, TbNfs is essential for tRNA thiolation in T. brucei (J Lukeš and JD Alfonzo, in prep.). Indeed, reduction in the expression levels of TbNfs leads to the expected reduction in thiolation of tRNA ${ }^{\mathrm{Glu}}$ and $\mathrm{tRNA}^{\mathrm{Gln}}$ but, significantly, causes no defect in tRNA distribution. Based on this result, we postulate that, rather unexpectedly, the use of thiolation as a negative import determinant is not conserved between the closely related genera Trypanosoma and Leishmania. TbNfs also serves as a key enzyme in Fe-S clusters synthesis pathway (Lill and Muehlenhoff 2008). Since the Rieske protein contains several Fe-S clusters, the lack of effect of the TbNfs knockdown on tRNA import also brings into question the essentiality of the Rieske protein for this process. Thus, in line with results obtained with the TbNfs knockdown cells, down-regulation of the Rieske protein levels by RNAi had no effect on tRNA import in vivo and/ or in vitro in T. brucei.

It was shown earlier in T. brucei that the Rieske subunit of respiratory complex III is essential for both maintenance of membrane potential and respiration (Horváth et al. 2005). Our current findings that the Rieske protein is not involved in tRNA import also firmly established that neither process plays a major role in mitochondrial tRNA localization and corroborates previous observations 


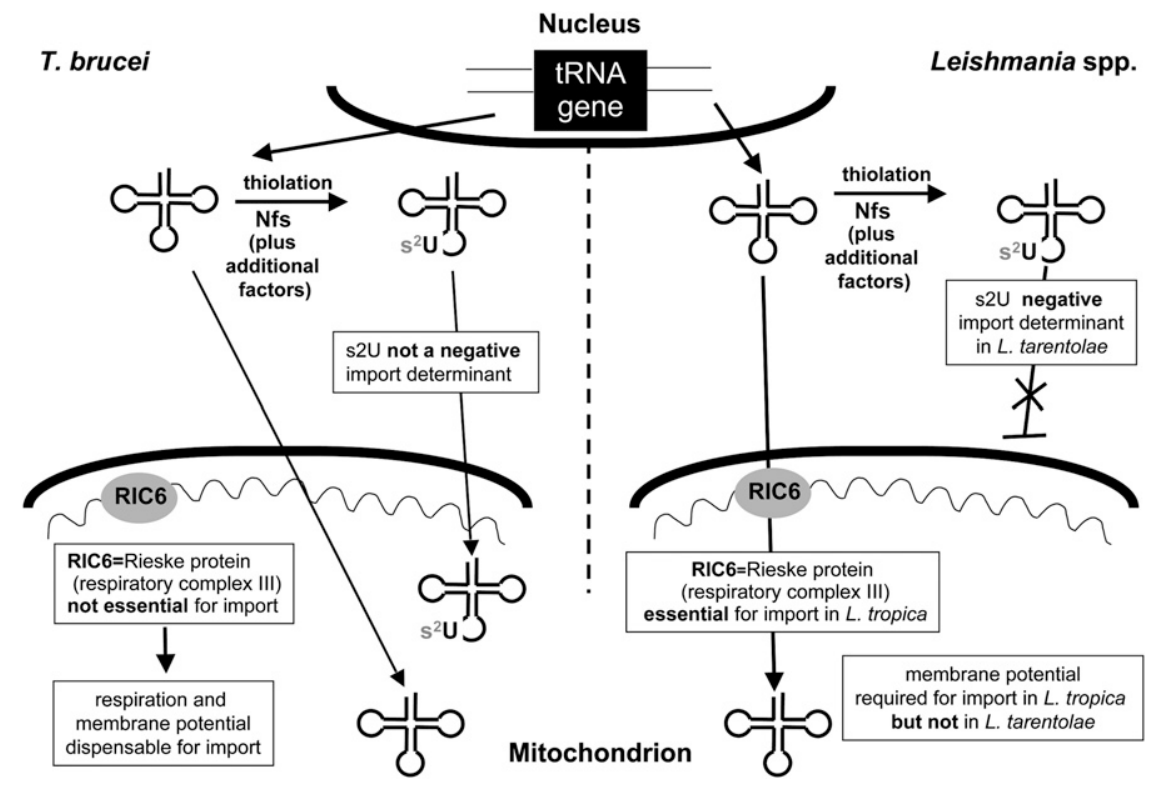

FIGURE 6. A model for the import of tRNAs into T. brucei mitochondria. All mitochondrial tRNAs are encoded in the nuclear genome and imported to the mitochondria from the cytosol. The cysteine desulferase (TbNfs), part of the iron sulfur clusters (ISC) assembly pathway, is the key enzyme for $\mathrm{s}^{2} \mathrm{U}$ formation in vivo in both the cytoplasm and the mitochondria ( $\mathrm{J}$ Lukes and JD Alfonzo, in prep.). The $\mathrm{s}^{2} \mathrm{U}$ modification (2-thiolation) at the anticodon wobble position could act as a negative determinant for tRNA import in L. tarentolae (Kaneko et al. 2003) but is not a negative determinant for import in T. brucei. The Rieske $\mathrm{Fe}-\mathrm{S}$ protein (respiratory complex III) is an essential component of the RIC in L. tropica (Mukherjee et al. 2007). This same protein does not play a significant role in tRNA import into T. brucei mitochondria. Because of its requirement for respiration and maintenance of a membrane potential neither process is critical for import as previously suggested through in vitro import assays in T. brucei and L. tarentolae.

(Nabholz et al. 1999; Rubio et al. 2000). Remarkably, the lack of a requirement for membrane potential is a conserved feature of the T. brucei and $L$. tarentolae pathways (Nabholz et al. 1999; Rubio et al. 2000) but differs from what has been shown in L. tropica (Fig. 6; Bhattacharyya and Adhya 2004). Overall, the data presented here demonstrate surprising differences between tRNA import systems even between relatively closely related organisms. Identification of both common and unique components of the tRNA import machineries will shed light on the origin and evolutionary constraints of this key pathway for a compartmentalized cell (Fig. 6).

\section{MATERIALS AND METHODS}

\section{Cell growth, RNAi induction, and purification of mitochondria}

The procyclic T. brucei 29-13 strains were constructed for inducible RNAi against either the cysteine desulfurase TbNfs (=TbIscS2) (Smid et al. 2006) or the Rieske protein (Horváth et al. 2005) using the pZJM or p2T7-177 vectors, respectively. Both wild-type $29-13$ procyclics, as well as the RNAi cell lines, were grown in SDM-79 medium supplemented with $10 \%$ fetal bovine serum (PAA), $7.5 \mu \mathrm{g} / \mathrm{mL}$ hemin (Sigma), and antibiotics as needed (50 $\mu \mathrm{g} / \mathrm{mL}$ hygromycin, $15 \mu \mathrm{g} /$ $\mathrm{mL}$ neomycin, and $2.5 \mu \mathrm{g} / \mathrm{mL}$ phleomycin). Synthesis of double-stranded (ds) RNA was induced by the addition of $1 \mu \mathrm{g} / \mathrm{mL}$ tetracycline. Growth rates of the noninduced and RNAi-induced clonal cell lines were determined every $24 \mathrm{~h}$ using the Beckman $\mathrm{Z} 2$ Coulter counter over a period of $8 \mathrm{~d}$. Mitochondrial vesicles were isolated from the noninduced and RNAi-induced cells by hypotonic lysis following protocols described elsewhere (Kapushoc et al. 2000).

\section{Immunoblot analysis}

Cell lysates of $T$. brucei procyclics corresponding to $5 \times 10^{6}$ cells/lane were resolved on $12 \%$ SDS-polyacrylamide gel. The polyclonal rabbit antibodies against the T. brucei TbNfs and Rieske proteins were used as described previously (Horváth et al. 2005; Smid et al. 2006). Secondary anti-rabbit IgG antibodies (1:2000) coupled to horseradish peroxidase (Sevapharma) were visualized according to the manufacturer's protocol using the ECL kit (Pierce).

\section{tRNA localization using Northern blot analysis}

RNA was isolated using the guanidinium thiocyanate/phenol/chloroform extraction method (Chomczynski and Sacchi 1987) either from whole cells or from the mitochondrial fractions. Samples (2.5 $\mu \mathrm{g}$ each) were separated on denaturating $8 \%$ polyacrylamide gel with $8 \mathrm{M}$ urea and electroblotted to Zeta-probe (Bio-Rad) membranes, which were subsequently probed with $\left[{ }^{32} \mathrm{P}\right]-5^{\prime}$ end-labeled oligonucleotides specific for each RNA as follows:

Cys-GCA: 5'-GGGGACCATTCGGACTGC-3';

Gln-UUG: 5' -CAGGATTCGAACCTGGGTTTTCG-3';

Glu-UUC: 5'-TTCCGGTACCGGGAATCGAAC-3';

Gly-UCC: 5'-TCTCCCGCTTGGAAGGCGGAAATC-3';

Ile-UAU: 5'-GGGGTTCGAACCCGCGATATTCGGT-3';

Val-UAC: 5'-ACGTTCTGCGTGTAAAGCAGACATC-3';

12S rRNA: 5'-GCTGCTACTGGGAGCTTCTCATAC-3'; and

SLRNA: 5'-AGGAGAGTAGGACTTGCCCT-3'.

Hybridization procedures were carried out according to the manufacturer's instructions (Bio-Rad). Images were taken with a Storm PhosphorImager (Molecular Dynamics). Abundance of individual tRNA species, isolated from cells prior to or after RNAi induction, was calculated in percentages using the ImageQuant program after normalization to the 12S RNA signal.

\section{In vitro import assays}

For import assays, $1 \mathrm{mg}$ of purified T. brucei mitochondria was incubated with $50-100,000 \mathrm{cpm}$ of radioactively labeled tRNA, $0.25 \mathrm{M}$ sucrose, $20 \mathrm{mM}$ Tris- $\mathrm{HCl}$ ( $\mathrm{pH}$ 8.0), $1 \mathrm{mM}$ ATP, $2 \mathrm{mM}$ 
DTT, $10 \mathrm{mM} \mathrm{MgCl}_{2}$, and $2 \mathrm{mM}$ EDTA. After incubation at $27^{\circ} \mathrm{C}$ for $10 \mathrm{~min}, 100 \mathrm{U}$ of micrococcal nuclease and $5 \mathrm{mM} \mathrm{CaCl}_{2}$ were added, and the reaction was incubated an additional $30 \mathrm{~min}$ to digest the tRNAs that were not imported into the mitochondria. Reactions were then stopped by the addition of 10 mM EGTA ( $\mathrm{pH}$ 8.0). To purify the protected tRNAs, the mitochondria were washed with $0.25 \mathrm{M}$ sucrose $/ 20 \mathrm{mM}$ Tris- $\mathrm{HCl}$ ( $\mathrm{pH} 8.0)$, pelleted, suspended in $90 \mu \mathrm{l}$ of $10 \mathrm{mM}$ Tris- $\mathrm{HCl}$ ( $\mathrm{pH} 8.0$ ), $1 \mathrm{mM}$ EDTA, and phenol extracted, followed by ethanol precipitation. The radioactively labeled tRNAs were separated by electrophoresis through a $7 \mathrm{M}$ urea/ $6 \%$ polyacrylamide gels. After electrophoresis, the gels were dried onto Whatman 3MM chromatography paper and visualized with the Storm PhosphorImager imaging system. Import assays were performed with either radioactive in vitro transcript representing the various tRNAs or with native tRNAs. Whenever native substrates were used, they were hybridized with an antisense biotinylated oligonucleotide specific for each species and purified by affinity chromatography through streptavidin beads as described elsewhere (Crain et al. 2002). Native tRNAs were $5^{\prime}$-end-labeled as described (Kaneko et al. 2003)

\section{ACKNOWLEDGMENTS}

This work was supported by a GM084065 grant from NIGMS and an MCB-0620707 grant from NSF to J.D.A. and the Grant Agency of the Czech Republic (204/06/1558) and the Czech Ministry of Education (LC07032 and 2B0612) to J.L. We also thank members of the Alfonzo and Lukeš laboratories for discussions, comments, and suggestions.

Received February 5, 2009; accepted April 13, 2009.

\section{REFERENCES}

Bhattacharyya SN, Adhya S. 2004. tRNA-triggered ATP hydrolysis and generation of membrane potential by the Leishmania mitochondrial tRNA import complex. J Biol Chem 279: 11259-11263.

Bouzaidi-Tiali N, Aeby E, Charriere F, Pusnik M, Schneider A. 2007. Elongation factor 1a mediates the specificity of mitochondrial tRNA import in T. brucei. EMBO J 26: 4302-4312.

Chomczynski P, Sacchi N. 1987. Single-step method of RNA isolation by acid guanidinium thiocyanate-phenol-chloroform extraction. Anal Biochem 162: 156-159.

Crain PF, Alfonzo JD, Rozenski J, Kapushoc ST, McCloskey JA, Simpson L. 2002. Modification of the universally unmodified uridine- 33 in a mitochondria-imported edited tRNA and the role of the anticodon arm structure on editing efficiency. RNA 8: 752-761.

Entelis N, Brandina I, Kamenski P, Krasheninnikov IA, Martin RP, Tarassov I. 2006. A glycolytic enzyme, enolase, is recruited as a cofactor of tRNA targeting toward mitochondria in Saccharomyces cerevisiae. Genes \& Dev 20: 1609-1620.

Esseiva AC, Naguleswaran A, Hemphill A, Schneider A. 2004. Mitochondrial tRNA import in Toxoplasma gondii. J Biol Chem 279: 42363-42368.

Haanstra JR, van Tuijl A, Kessler P, Reijnders W, Michels PA, Westerhoff HV, Parsons M, Bakker BM. 2008. Compartmentation prevents a lethal turbo-explosion of glycolysis in trypanosomes. Proc Natl Acad Sci 105: 17718-17723.

Hancock K, LeBlanc AJ, Donze D, Hajduk SL. 1992. Identification of nuclear encoded precursor tRNAs within the mitochondrion of Trypanosoma brucei. J Biol Chem 267: 23963-23971.

Horváth A, Horáková E, Dunajčíková P, Verner Z, Pravdová E, Šlapetová I, Cuninková L, Lukeš J. 2005. Downregulation of the nuclear-encoded subunits of the complexes III and IV disrupts their respective complexes but not complex I in procyclic Trypanosoma brucei. Mol Microbiol 58: 116-130.

Ikeuchi Y, Shigi N, Kato J, Nishimura A, Suzuki T. 2006. Mechanistic insights into sulfur relay by multiple sulfur mediators involved in thiouridine biosynthesis at tRNA wobble positions. Mol Cell 21: 97-108.

Kambampati R, Lauhon CT. 2000. Evidence for the transfer of sulfane sulfur from IscS to ThiI during the in vitro biosynthesis of 4-thiouridine in Escherichia coli tRNA. J Biol Chem 275: 10727-10730.

Kambampati R, Lauhon CT. 2003. MnmA and IscS are required for in vitro 2-thiouridine biosynthesis in Escherichia coli. Biochemistry 42: 1109-1117.

Kaneko T, Suzuki T, Kapushoc ST, Rubio MA, Ghazvini J, Watanabe K, Simpson L. 2003. Wobble modification differences and subcellular localization of tRNAs in Leishmania tarentolae: Implication for tRNA sorting mechanism. EMBO J 22: 657-667.

Kapushoc ST, Alfonzo JD, Rubio MA, Simpson L. 2000. End processing precedes mitochondrial importation and editing of tRNAs in Leishmania tarentolae. J Biol Chem 275: 37907-37914.

Lill R, Mühlenhoff U. 2008. Maturation of iron-sulfur proteins in eukaryotes: Mechanisms, connected processes, and diseases. Annu Rev Biochem 77: 669-700.

Lima BD, Simpson L. 1996. Sequence-dependent in vivo importation of tRNAs into the mitochondrion of Leishmania tarentolae. RNA 2: $429-440$.

Mahapatra S, Ghosh T, Adhya S. 1994. Import of small RNAs into Leishmania mitochondria in vitro. Nucleic Acids Res 22: 33813386.

Mahapatra S, Ghosh S, Bera SK, Ghosh T, Das A, Adhya S. 1998. The $\mathrm{D}$ arm of $\mathrm{tRNA}^{\mathrm{Tyr}}$ is necessary and sufficient for import into Leishmania mitochondria in vitro. Nucleic Acids Res 26: 20372041.

Marechal-Drouard L, Weil JH, Guillemaut P. 1988. Import of several tRNAs from the cytoplasm into the mitochondria in bean Phaseolus vulgaris. Nucleic Acids Res 16: 4777-4788.

Martin RP, Schneller JM, Stahl AJ, Dirheimer G. 1979. Import of nuclear deoxyribonucleic acid coded lysine-accepting transfer ribonucleic acid (anticodon C-U-U) into yeast mitochondria. Biochemistry 18: 4600-4605.

Mihara H, Kato S, Lacourciere GM, Stadtman TC, Kennedy RA, Kurihara T, Tokumoto U, Takahashi Y, Esaki N. 2002. The iscS gene is essential for the biosynthesis of 2-selenouridine in tRNA and the selenocysteine-containing formate dehydrogenase H. Proc Natl Acad Sci 99: 6679-6683.

Mottram JC, Bell SD, Nelson RG, Barry JD. 1991. tRNAs of Trypanosoma brucei. Unusual gene organization and mitochondrial importation. J Biol Chem 266: 18313-18317.

Mukherjee S, Basu S, Home P, Dhar G, Adhya S. 2007. Necessary and sufficient factors for the import of transfer RNA into the kinetoplast mitochondrion. EMBO Rep 8: 589-595.

Nabholz CE, Horn EK, Schneider A. 1999. tRNAs and proteins are imported into mitochondria of Trypanosoma brucei by two distinct mechanisms. Mol Biol Cell 10: 2547-2557.

Nakai Y, Umeda N, Suzuki T, Nakai M, Hayashi H, Watanabe K, Kagamiyama H. 2004. Yeast Nfs1p is involved in thio-modification of both mitochondrial and cytoplasmic tRNAs. J Biol Chem 279: 12363-12368.

Nilsson K, Lundgren HK, Hagervall TG, Bjork GR. 2002. The cysteine desulfurase IscS is required for synthesis of all five thiolated nucleosides present in tRNA from Salmonella enterica serovar typhimurium. J Bacteriol 184: 6830-6835.

Pusnik M, Charriere F, Maser P, Waller R, Dagley MJ, Lithgow T, Schneider A. 2009. The single mitochondrial porin of Trypanosoma brucei is the main metabolite transporter in the outer mitochondrial membrane. Mol Biol Evol 26: 671-680.

Rinehart J, Krett B, Rubio MA, Alfonzo JD, Soll D. 2005. Saccharomyces cerevisiae imports the cytosolic pathway for Gln-tRNA synthesis into the mitochondrion. Genes \& Dev 19: 583-592. 


\section{Paris et al.}

Rubio MA, Liu X, Yuzawa H, Alfonzo JD, Simpson L. 2000. Selective importation of RNA into isolated mitochondria from Leishmania tarentolae. RNA 6: 988-1003.

Rubio MA, Rinehart JJ, Krett B, Duvezin-Caubet S, Reichert AS, Soll D, Alfonzo JD. 2008. Mammalian mitochondria have the innate ability to import tRNAs by a mechanism distinct from protein import. Proc Natl Acad Sci 105: 9186-9191.

Rusconi CP, Cech TR. 1996a. The anticodon is the signal sequence for mitochondrial import of glutamine tRNA in Tetrahymena. Genes \& Dev 10: $2870-2880$.

Rusconi CP, Cech TR. 1996b. Mitochondrial import of only one of three nuclear-encoded glutamine tRNAs in Tetrahymena thermophila. EMBO J 15: 3286-3295.

Salinas T, Duchene AM, Delage L, Nilsson S, Glaser E, Zaepfel M, Marechal-Drouard L. 2006. The voltage-dependent anion channel, a major component of the tRNA import machinery in plant mitochondria. Proc Natl Acad Sci 103: 18362-18367.

Salinas T, Duchene AM, Marechal-Drouard L. 2008. Recent advances in tRNA mitochondrial import. Trends Biochem Sci 33: 320-329.

Schmitz UK, Lonsdale DM. 1989. A yeast mitochondrial presequence functions as a signal for targeting to plant mitochondria in vivo. Plant Cell 1: 783-791.

Schneider A, Marechal-Drouard L. 2000. Mitochondrial tRNA import: Are there distinct mechanisms? Trends Cell Biol 10: 509-513.

Simpson AM, Suyama Y, Dewes H, Campbell DA, Simpson L. 1989. Kinetoplastid mitochondria contain functional tRNAs which are encoded in nuclear DNA and also contain small minicircle and maxicircle transcripts of unknown function. Nucleic Acids Res 17: $5427-5445$

Smid O, Horakova E, Vilimova V, Hrdy I, Cammack R, Horvath A, Lukeš J, Tachezy J. 2006. Knock-downs of iron-sulfur cluster assembly proteins IscS and IscU down-regulate the active mitochondrion of procyclic Trypanosoma brucei. J Biol Chem 281: 28679-28686.

Suyama Y. 1986. Two-dimensional polyacrylamide gel electrophoresis analysis of Tetrahymena mitochondrial tRNA. Curr Genet 10: 411420.

Suyama Y, Wong S, Campbell DA. 1998. Regulated tRNA import in Leishmania mitochondria. Biochim Biophys Acta 1396: 138142.

Tarassov IA, Martin RP. 1996. Mechanisms of tRNA import into yeast mitochondria: An overview. Biochimie 78: 502-510.

Tarassov I, Entelis N, Martin RP. 1995a. An intact protein translocating machinery is required for mitochondrial import of a yeast cytoplasmic tRNA. J Mol Biol 245: 315-323.

Tarassov I, Entelis N, Martin RP. 1995b. Mitochondrial import of a cytoplasmic lysine-tRNA in yeast is mediated by cooperation of cytoplasmic and mitochondrial lysyl-tRNA synthetases. EMBO J 14: $3461-3471$.

Yermovsky-Kammerer AE, Hajduk SL. 1999. In vitro import of a nuclearly encoded tRNA into the mitochondrion of Trypanosoma brucei. Mol Cell Biol 19: 6253-6259. 

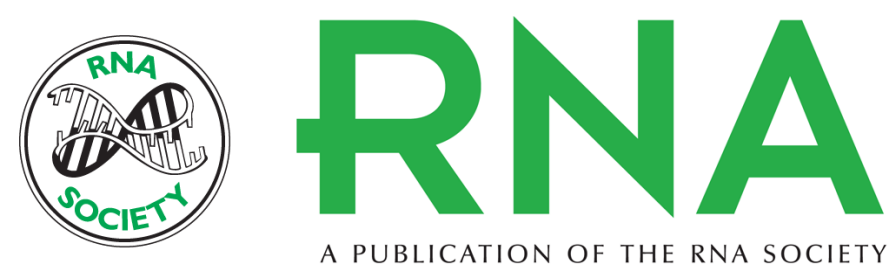

A PUBLICATION OF THE RNA SOCIETY

\section{Mitochondrial tRNA import in Trypanosoma brucei is independent of thiolation and the Rieske protein}

Zdenek Paris, Mary Anne T. Rubio, Julius Lukes, et al.

RNA 2009 15: 1398-1406 originally published online May 22, 2009

Access the most recent version at doi:10.1261/rna.1589109

$\begin{array}{ll}\text { References } & \begin{array}{l}\text { This article cites } 44 \text { articles, } 23 \text { of which can be accessed free at: } \\ \text { http://rnajournal.cshlp.org/content/15/7/1398.full.html\#ref-list-1 }\end{array}\end{array}$

License

Email Alerting Receive free email alerts when new articles cite this article - sign up in the box at the Service top right corner of the article or click here.

To subscribe to $R N A$ go to:

http://rnajournal.cshlp.org/subscriptions 